\title{
Self-paced EEG-based Brain-controlled car in Real-World Enviroment
}

\author{
Yang $\mathrm{Yu}^{\mathrm{a}}$, Zongtan Zhou ${ }^{\mathrm{b}}$, Jun Jiang ${ }^{\mathrm{c}}$, Yadong Liu ${ }^{\mathrm{d}}$, and Dewen $\mathrm{Hu}^{\mathrm{e}^{\star}}$ \\ College of Mechatronic Engineering and Automation, National University of Defense Technology, \\ Changsha, Hunan, 410073, China \\ ayuyangnudt@hotmail.com, ${ }^{\mathrm{b}}$ narcz@163.com, cjiangjun@nudt.edu.cn, dliuyadong1977@163.com, \\ $\mathrm{e}^{*} \mathrm{dwhu@nudt.edu.cn}$
}

Keywords: Brain-computer interface, Electroencephalogram (EEG), Motor imagery, Asynchronous protocol, Brain-actuated car.

\begin{abstract}
This study presented the design and online experiments of a self-paced EEG-based BCI for controlling a car in real world environment. This paradigm using two distinct MI tasks to generate a multi-task car control strategy, including start, move forwards, turn left, turn right, move backwards, and stop. The experiment results suggested that a brain-actuated car is possible when it runs in low velocity, approximately $5 \mathrm{~km} /$ hour in our study. This proposed self-paced EEG-based BCI paradigm could potentially help disabled and paralyzed people to gain more mobility in the future, and moreover, provide a supplementary car driving strategy to healthy people.
\end{abstract}

\section{Introduction}

Brain-computer interface (BCI) techniques have developed rapidly in the pasting two decades. A BCI is a communication system in which an individual can send commands to the external word by generating specific patterns in brain signals without depending on brain's output channels of peripheral nerves and muscles [1]. The main purpose for BCIs is to enable communication for people with severe disabilities and neurological conditions [2]. BCI can provide direct access to brain states and therefore, enable improved man-machine interaction for healthy people [3, 4]. And various studies have explored the potential of BCI applications in many fields: communication [5], neuroprosthetics [6, 7], robots [8-10], mobility control [11, 12]. These previous studies have promisingly demonstrated the possibility of BCIs for controlling external devices.

Bring the BCIs from the laboratory conditions to real world applications is an important aspect for $\mathrm{BCI}$ researching. In the current study, we proposed an asynchronous EEG-based BCI paradigm using two distinct MI tasks to generate a multi-task car control strategy, including start, move forwards, turn left, turn right, move backwards, and stop. To hit our targets, two classifiers need to be trained. Firstly, a control intention classier (CIC) was trained to determine whether the ongoing signal represented rest or MI tasks. Secondly, a left/right classier (LRC) was trained to distinguish between left/right MI tasks.

\section{Methods}

System introduction. The BCI control system mainly included three subsystems: BCI signal processing system, and the car control system. The BCI signal processing system records and analyzes the EEG signals and translates them into control commands. The interface system presents the car states and monitors the commands sent to the car. The car control system receives control commands and converts them into electric signals to drive the car (Fig. 1). 


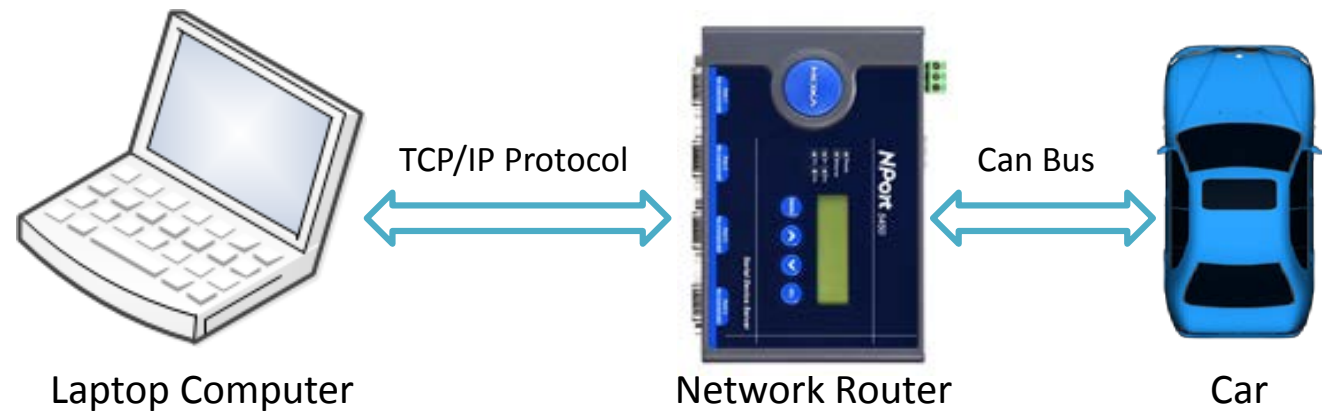

Fig. 1 Car control system.

Participants. Four healthy subjects who have already got their driving licenses (males, age 22-26 years, with an average age of 24 years) participated in the study, all native Chinese speakers. And none had any known cognitive deficit. Two of the subjects had previous MI BCI experiences, and the other subject was complete novices. All of the subjects signed informed consent to participant after receiving a complete description of this study.

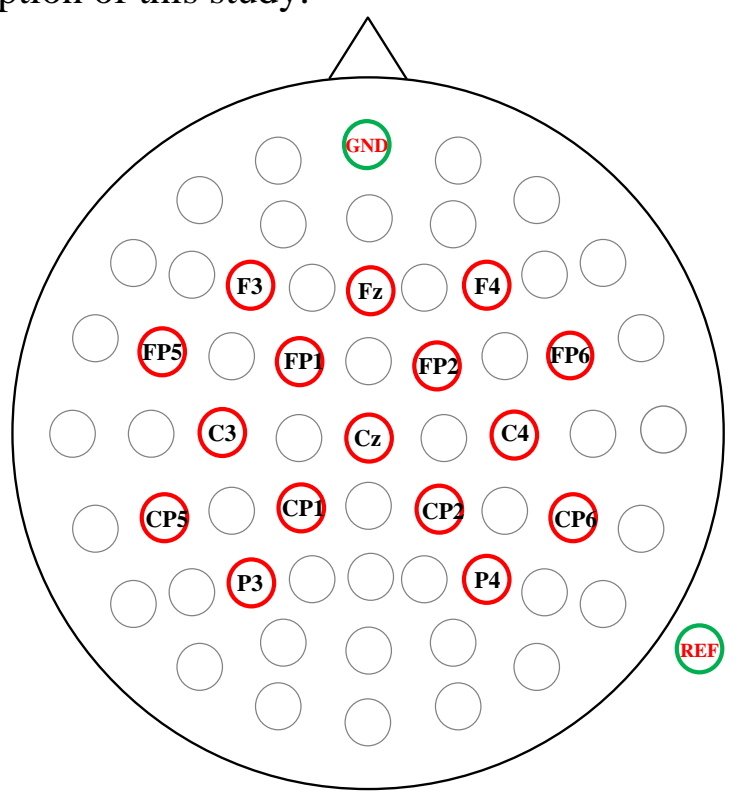

Fig. 2 EEG electrode positions based on the 64-channel modified international 10-20 system.

EEG data acquisition and preprocessing. EEG signals were recorded using an EEG cap embedded with 16 electrodes around the sensorimotor cortex (C3, Cz, C4, F3, Fz, F4, P3, P4, CP1, CP2, CP5, CP6, FC1, FC2, FC5 and FC6), based on the 64-channel modified international 10-20 system. The electrode montage is depicted in Figure 2. Each channel was referenced to P8 and grounded to FPz. The electrode impedance was maintained below $5 \mathrm{k} \Omega$. The EEG signals were amplified using a BrainAmp DC Amplifier (Brain Products GmbH, Germany), digitized at a rate of $200 \mathrm{~Hz}$, and filtered using a $50 \mathrm{~Hz}$ notch filter. The stimulus presentation, data collection, offline signal processing, and experimental procedures were conducted using the BCI2000 framework [13], which provides a Python interface for stimuluspresentation and a Matlab interface for signal processing.

Classification. The EEG signals were spatially filtered using common spatial pattern (CSP), and then linear discriminant analysis (LDA) was used to solve the classification problems. Calibration of the two classifiers coefficients was performed offline using the data collected in the calibration experiments.

\section{Experimental protocol}

The offline experiments. During the offline experimentation, the subjects were asked to sit relax in the driving seat in the car and to avoid overt eye/body movements other than requested. The design and purpose of the experiment were explained to each subject in detail, and a short familiarization 
session was performed prior to the initiation of the experiments. The experimental procedure was conducted in a laptop placed in the backseat, and the Turn-Lamps of the car were controlled by this laptop using TCP/IP and can-bus protocol and therefore they could prompt the participants to execute the exact MI task. Each session consisted of 15 trials per MI task, and each MI task was performed four seconds in a random order with a four-second break between two trials as rest state, during which time the participants were asked to keep calm and avoid mental imagination.

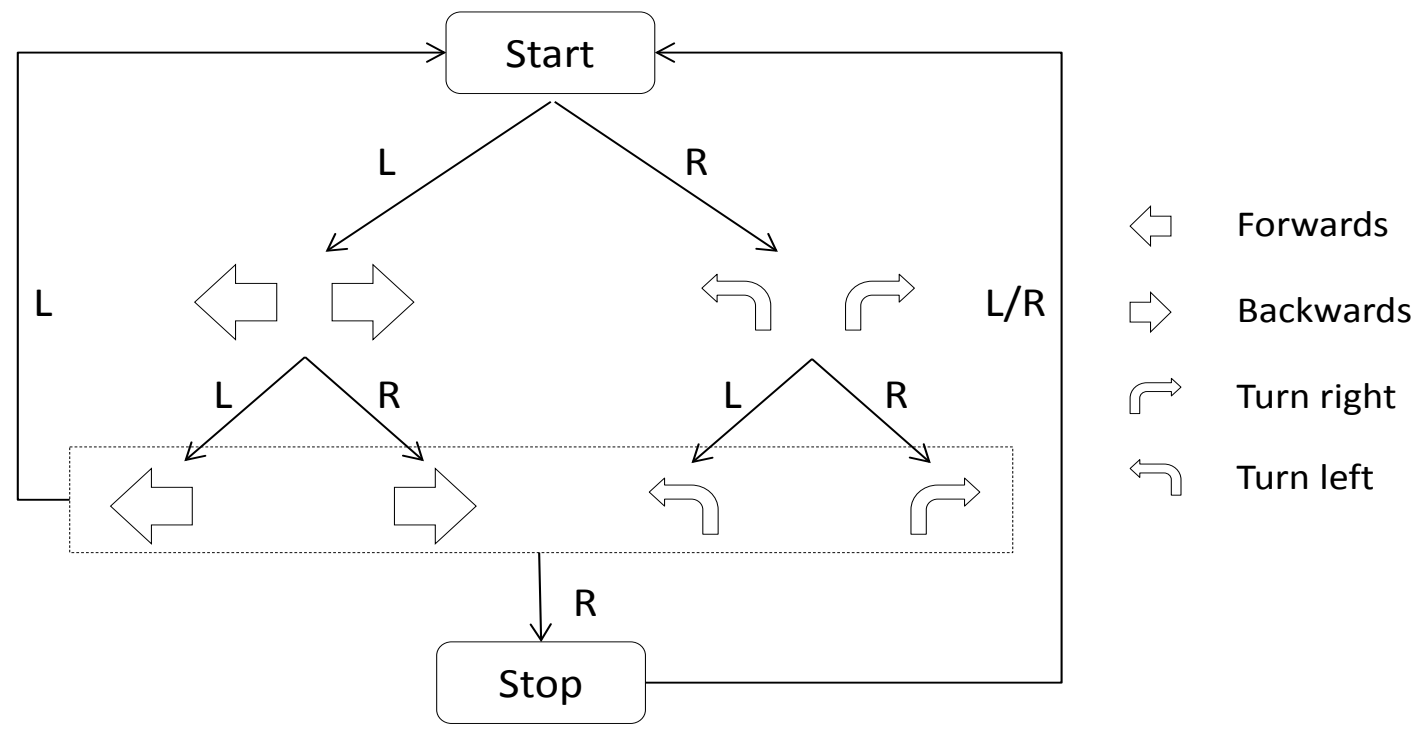

Fig. 3 Diagram of the car control protocol.

The online car control experiment. During online car control experiment, the ongoing EEG signals were firstly subjected to the CIC to extract the participant's mental state (Rest-state or MI-state), If MI-state was detected, then the LRC classified it into left or right MI. Fig. 3 showed the diagram of the control protocols. The performance of the BCI system was evaluated via calculating the classification accuracy of the two MI tasks and the information transfer rate (ITR) [2]. In order to test the control performance of our paradigm, two participants was asked to drive the car following the route previously lined out (A-B-C), as show in Fig. 4.

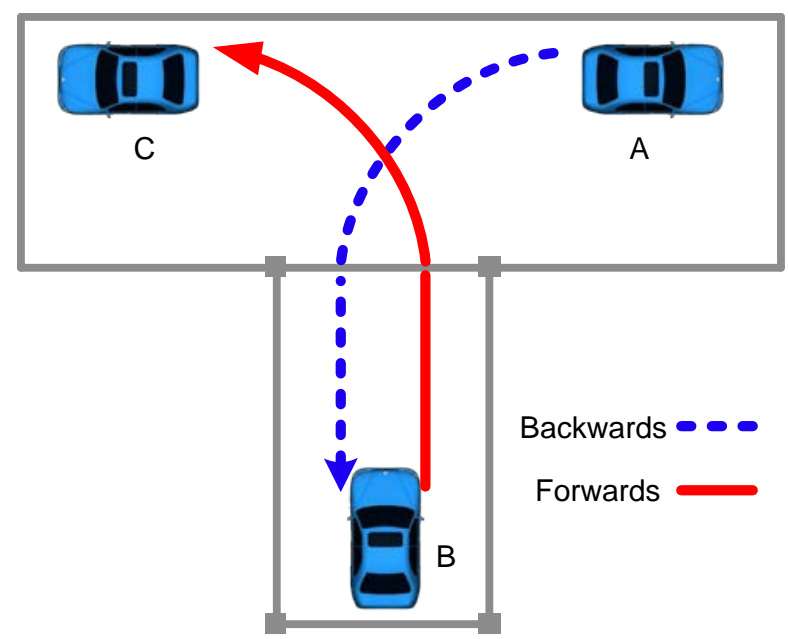

Fig. 4 online car control. the task of the subject was to control the car move backwards from A to B, and then move forwards from B to C.

\section{Results and Conclusion}

All the subjects participated in the offline training experiment for left/right MI tasks, and the classification accuracies are shown in Table 1. Two participants joined the online control experiment. the total time, and the number of commands taken to accomplish one task are illustrated in Table 1. 
Table 1 Offline classification and online control performance.

\begin{tabular}{ccccc}
\hline Subject & \multicolumn{2}{c}{ Offline Experiment } & \multicolumn{2}{c}{ Online Experiment } \\
& Accuracy(L/R,\%) & ITR(bit/min) & Time (min) & Commands \\
\cline { 2 - 5 } Sub1 & $87.4 / 89.8$ & 15.21 & 8.12 & 14 \\
Sub2 & $85.1 / 84.2$ & 12.83 & 11.40 & 20 \\
Sub3 & $78.9 / 77.6$ & 11.10 & -- & -- \\
Sub4 & $75.4 / 71.3$ & 10.15 & -- & -- \\
\hline
\end{tabular}

The experiment results suggested that a brain-actuated car is possible when it runs in low velocity, approximately 5km/hour in our study. This proposed self-paced EEG-based BCI paradigm could potentially help disabled and paralyzed people to gain more mobility in the future, and moreover, provide a supplementary car driving strategy to healthy people.

\section{Acknowledgments}

This work is supported by the National Natural Science Foundation of China (Grant No 61375117, and 91320202, and 91420302).

\section{Literature References}

[1] Tsui CSL, Gan JQ, Roberts SJ. A self-paced brain-computer interface for controlling a robot simulator: an online event labelling paradigm and an extended Kalman filter based algorithm for online training. Med Biol Eng Comput. 2009;47:257-65.

[2] Wolpaw JR, Birbaumer N, McFarland DJ, Pfurtscheller G, Vaughan TM. Brain-computer interfaces for communication and control. Clinical Neurophysiology. 2002;113:767-91.

[3] Wolpaw J, McFarland D. Control of a two-dimensional movement signal by a noninvasive brain-computer interface in humans. Proc Natl Acad Sci 2004;101(51):17849-54.

[4] Wolpaw J, Birbaumer N, Heetderks WJ, McFarland DJ, H.Peckham P, Schalk G, et al. Brain-computer interface technology: A review of the first international meeting. 2000;8(2):164-73.

[5] Pan J, Li Y, Gu Z, Yu Z. A comparison study of two P300 speller paradigms for brain-computer interface. Cogn Neurodyn. 2013(7):523-9.

[6] Rebsamen B, Guan C, Zhang H, Wang C, Teo C, Marcelo H. Ang J, et al. A Brain Controlled Wheelchair to Navigate in Familiar Environments. IEEE Trans Neural Syst Rehabil Eng,. 2010;18:590-8.

[7] R. Leeb DF, Muller-Putz GR, Scherer R, Slater M, Pfurtscheller G. Self-paced (asynchronous) BCI control of a wheelchair in virtual environments: A case study with a tetraplegic,. Comput Intell Neurosci. 2007:796421-8.

[8] Galan F, Nuttin M, Lew E, Ferrez PW, Vanacker G, Philips J, et al. A brain-actuated wheelchair: Asynchronous and non-invasive Brain-computer interfaces for continuous control of robots. Clinical Neurophysiology. 2008(119):2159-69.

[9] Chae Y, Jeong J, Jo S. Toward Brain-Actuated Humanoid Robots: Asynchronous Direct Control Using an EEG-Based BCI. IEEE Trans Robot. 2012;25:11131-1144.

[10] Millan J, Renkens F, Mourino J, Gerstner W. Noninvasive brainactuated control of a mobile robot by human EEG. IEEE Trans Biomed Eng. 2004;51:1026-33.

[11]Rebsamen B, Burdet E, Guan C, Zhang H, Teo CL, Zeng Q, et al. A brain-controlled wheelchair based on P300 and path guidance. Int Conf Biomed Robot Biomechatron. 2006:1101-6.

[12] Iturrate I, Antelis J, Kubler A, Minguez J. A noninvasive brainactuated wheelchair based on a P300 neurophysiological protocol and automated navigation. IEEE Trans Robot. 2009;25(3):614-27.

[13] Schalk G, McFarland DJ, Hinterberger T, Birbaumer N, Wolpaw JR. BCI2000: a general-purpose brain-computer interface (BCI) system. IEEE Trans Biomed Eng 2004 (51):103443. 